\title{
Effect of high-intensity circuit training on obesity indices, physical fitness, and browning factors in inactive female college students
}

\author{
Jun-Soo Lee', Eun-Sun Yoon², Sun-Young Jung ${ }^{3}$, Ki-Tae Yim', Dae-Young Kim, ${ }^{1, * *}$ \\ IInstitute of Digital Anti-Aging Healthcare, Inje University, Gimhae, Korea \\ 2Department of Sports for All, Korea National Open University, Seoul, Korea \\ ${ }^{3}$ Department of Physical Therapy, Hosan University, Gyeongsan, Korea \\ ${ }^{4}$ College of Creative Future Talent, Daejin University, Pocheon, Korea \\ ${ }^{5}$ Department of Sports Healthcare, College of Social Sciences, Inje University, Gimhae, Korea
}

The recently discovered myokines, irisin and fibroblast growth factor-21, have emerged beneficial for metabolic health due to their role in regulating browning. This study examined the effects of high-intensity circuit training on obesity indices, physical fitness, and irisin and fibroblast growth factor-21 levels in inactive female college students. Ten female college students performed high-intensity circuit training ljogging, stretching, squat jumps, arm walking and push-ups, lunge jumps, burpee test, mountain climbers, side steps, and crunches and side crunches exercises). The exercise program consisted of $40 \mathrm{~min}$ of circuit training at $60 \%-80 \%$ hear rate reserve and was conducted 3 times per week for 4 weeks. The body composition, physical fitness, and concentration of plasma irisin and fibroblast growth factor-21 were ana- lyzed before and after the exercise. The body weight $(P=0.001)$, waist circumference $(P=0.003)$, and body fat percentage $(P=0.003)$ decreased, while the muscular strength (handgrip strength test, $P=0.030$; sit-ups test, $P=0.024)$ and cardiorespiratory fitness $(P=0.001)$ increased after the exercise program. However, there were no significant changes in the irisin and fibroblast growth factor-21 levels. These results suggest that high-intensity circuit training could be the ideal type of exercise in inactive female college students to induce a positive change in the obesity indices and physical fitness. Further studies are needed to determine the effects of exercise on the browning factors.

Keywords: Exercise, Circuit training, Irisin, Fibroblast growth factor-21

\section{INTRODUCTION}

Because the overall health management during the undergraduate years has an impact on the entire adulthood, physical activity in this period is critical (Lackman et al., 2015). Currently, female college students in their 20s show a tendency to control their weight through extreme diet control rather than regular exercise, because they perceive the skinny body type as the ideal appearance and lack the time to perform exercises due to the high academic demands. Such weight control techniques can lead to numerous health issues in the future such as metabolic and musculoskeletal diseases (Donaldson and Gordon, 2015; Heilbronn et al., 2007).
Recently, high-intensity circuit training (CT) has attracted interest as an exercise program providing various health benefits by utilizing relatively short time and limited efforts. CT consists of a short series of aerobic and resistance exercises performed in repetition. It is known to be efficient in developing both muscular strength and endurance (Klika and Jordan, 2013). Compared to low-intensity CT, the energy consumption per unit time is higher in high-intensity $\mathrm{CT}$ with high additional energy consumption during the resting period; hence, it is preferred by individuals who find it difficult to perform regular exercise due to the lack of time (Paoli et al., 2012). It is effective in improving the physical fitness with respect to the muscular strength, flexibility, and car-

\footnotetext{
${ }^{*}$ Corresponding author: Dae-Young Kim (D) https://orcid.org/0000-0002-4662-4463 Department of Sports Health Care, College of Social Sciences, Inje University, 197 Inje-ro, Gimhae 50834, Korea 
diorespiratory fitness, as well as in weight loss. Furthermore, it is more interesting than performing repetitive aerobic exercises and more enjoyable because of its sophisticated design that combines both aerobic and resistance exercises (Paoli et al., 2010; Skidmore et al., 2012).

Myocytes produce various myokines that regulate the metabolism along with other tissues including adipose tissue, liver, and pancreas (Pedersen et al., 2007). The recently discovered myokines, irisin and fibroblast growth factor-21 (FGF-21), are known to influence the metabolism rate by increasing the lipid metabolism and energy consumption (Giralt et al., 2015; Xiong et al., 2015). Irisin can activate energy consumption through the browning of white adipose tissue, leading to heat generation, weight control, and blood glucose homeostasis (Boström et al., 2012). FGF-21 increases the energy availability by promoting the use of glucose from the adipose and muscular tissues (Hojman et al., 2009) and plays a role in fatty acid oxidation and degradation (Potthoff et al., 2009) and adipogenesis inhibition (Coskun et al., 2008). In a previous study by Boström et al. (2012) on human subjects, the level of irisin significantly increased by approximately 2-fold after a 10-week moderate-intensity aerobic exercise program. Subsequently, studies have attempted to verify the positive effect of exercise on irisin and FGF-21 levels, but the results have been inconsistent and without any definite conclusion. Such discrepancies among the studies could be due to the variations in the participants' characteristics (age, level of obesity, level of physical fitness, etc.), exercise type and intensity, duration of exercise, and initial level of myokines (Fox et al., 2018). Acute high-intensity exercise has been shown to significantly increase irisin and FGF21 concentrations. Tsuchiya et al. (2014) reported that acute high-intensity exercise causes greater irisin responses compared with low-intensity exercise in young male adults. Also, Kim et al. (2013) reported similar result that high-intensity exercise increases FGF-21 in healthy young adult.

The findings of previous studies collectively suggest that high-intensity exercise is more effective than low-intensity exercise in increasing the levels of irisin and FGF-21. However, although previous finding showed grater increase of irisin an FGF21 via high-intensity exercise, such reports are lacking in female young adults. Thus, this study aimed to verify the effect of a short-term high-intensity CT exercise program on the physical fitness and irisin and FGF-21 browning factor levels in inactive female college students in their 20s.
Table 1. Physical characteristics of the subjects $(n=10)$

\begin{tabular}{lr}
\hline Characteristic & \multicolumn{1}{c}{ Mean \pm SD } \\
\hline Age $(\mathrm{yr})$ & $21.00 \pm 1.00$ \\
Height $(\mathrm{cm})$ & $159.17 \pm 7.23$ \\
Weight $(\mathrm{kg})$ & $63.48 \pm 9.01$ \\
Body fat $(\%)$ & $30.46 \pm 3.95$ \\
Body mass index $\left(\mathrm{kg} / \mathrm{m}^{2)}\right.$ & $25.14 \pm 2.51$ \\
\hline
\end{tabular}

SD, standard deviation.

\section{MATERIALS AND METHODS}

\section{Research participants}

This study included 10 female college students aged 20-23 years, who did not have any medical conditions and did not perform regular exercise. Participants were recruited from the Inje University and advertisement was placed on the University notice board. The purpose and contents of the study were adequately explained to all the subjects in advance, and the voluntary participants providing signed informed consent were included in this study. The experimental procedure was approved by the Ethics Committee of the Inje University (INJE2019-05-006). The characteristics of the subjects are summarized in Table 1.

\section{Exercise program}

The exercise program used in this study was high-intensity CT performed 3 times a week for 4 weeks in total. The exercise intensity was set as a score of 13-14 on the rating of perceived exertion (RPE) scale and 60\%-70\% heart rate reserve (HRR) for the first 2 weeks, followed by a slight increase to 14-18 RPE scale score and 65\%-80\% HRR in the subsequent 2 weeks. To monitor the heart rate during exercise, the POLAR heart rate monitor (Polar M400, Polar Electro, Espoo, Finland) was used. The time of a single round of exercise was set at $40 \mathrm{~min}$, which included the warmup (5 min), main exercise (30 min), and cool-down ( $5 \mathrm{~min})$. The exercise program comprised aerobic exercises and muscular strength exercises in a balanced combination to facilitate the use of the global muscles. Each exercise was performed for $30 \mathrm{sec}$ followed by $15 \mathrm{sec}$ of rest, and three sets were performed with $1 \mathrm{~min}$ break between the sets. The details of the exercise program are shown in Table 2.

\section{Analysis categories and methods}

All the measurable variables were measured at the same time both before and after the exercise to analyze its effect. To exclude any temporary effects of the exercise, postexercise measurements 
Table 2. High-intensity circuit exercise program

\begin{tabular}{|c|c|c|c|c|}
\hline Exercise stage & Program & Intensity & Time & Week \\
\hline \multirow[t]{2}{*}{ Warm-up } & - Jogging & \multirow{2}{*}{\multicolumn{3}{|c|}{$5 \min$}} \\
\hline & - Stretching & & & \\
\hline \multirow[t]{18}{*}{ Main exercise } & - Squats & $60 \%-70 \%$ & \multirow{9}{*}{$\begin{array}{c}\text { 30-sec exercise; } \\
15 \text { sec rest/set } \\
3 \text { sets } \\
30 \text { min }\end{array}$} & \multirow[t]{9}{*}{$1-2$} \\
\hline & •Arm walking & HRR & & \\
\hline & - Lunges & (RPE 13-14) & & \\
\hline & - Burpee test & & & \\
\hline & $\begin{array}{l}\text { - Mountain } \\
\text { climbers }\end{array}$ & & & \\
\hline & - Side step & & & \\
\hline & - Crunches & & & \\
\hline & • Jogging & & & \\
\hline & - Stretching & & & \\
\hline & - Squat Jumps & \multirow{9}{*}{$\begin{array}{c}65 \%-80 \% \\
\text { HRR } \\
\text { (RPE 14-18) }\end{array}$} & \multirow{9}{*}{$\begin{array}{c}\text { 30-sec exercise; } \\
15 \text { sec rest/set } \\
3 \text { sets }\end{array}$} & \multirow[t]{9}{*}{$3-4$} \\
\hline & $\begin{array}{c}\text { •Arm walking } \\
\text { \& push-ups }\end{array}$ & & & \\
\hline & - Lunge jumps & & & \\
\hline & - Burpee test & & & \\
\hline & $\begin{array}{c}\text { - Mountain } \\
\text { climbers }\end{array}$ & & & \\
\hline & - Side step & & & \\
\hline & $\begin{array}{l}\text { - Crunches \& } \\
\text { side crunches }\end{array}$ & & & \\
\hline & - Jogging & & & \\
\hline & - Stretching & & & \\
\hline Cool-down & - Stretching & & $5 \min$ & \\
\hline
\end{tabular}

HRR, heart rate reserve; RPE, rating of perceived exertion.

were conducted $72 \mathrm{hr}$ after the last round of exercise.

\section{Body composition}

The height $(\mathrm{cm})$ and weight $(\mathrm{kg})$ of each subject was measured using an automated analyzer (DS-102, Jenix, Seoul, Korea), and the body mass index (BMI) was then calculated using the formula "BMI = weight $(\mathrm{kg}) /$ height $(\mathrm{m})^{2}$." To measure the body fat percentage (BF\%), a body composition analyzer (Inbody 720, Inbody, Seoul, Korea) was used. A measuring tape was used to measure the waist circumference (WC) from the lowest part of the rib cage to the center of the iliac crest.

\section{Blood testing}

Blood tests were performed on all subjects after $12 \mathrm{hr}$ of fasting. The blood was drawn at the same time under the same conditions before and after the exercise. A disposable needle was used to draw $5 \mathrm{~mL}$ of venous blood from the anterior cardinal vein. The blood was stored in serum separating tubes for serum and centrifuged for $10 \mathrm{~min}$ at $3,000 \mathrm{rpm} ; 200 \mu \mathrm{L}$ of the serum was stored in a sample tube. The irisin and FGF-21 levels were assessed using the enzyme linked immunosorbent assay (ELISA) method with the irisin ELISA kit (BioVendor, Brno, Czech Republic) and FGF-21 Quantikine ELISA kit (R\&D System, Minneapolis, MN, USA), respectively.

\section{Physical fitness measurements}

The physical fitness in this study was estimated based on the muscular strength, muscular endurance, flexibility, and cardiorespiratory fitness. For muscular strength, a digital hand dynamometer (DW-781, Daewoosports Industry, Seoul, Korea) was used to assess the handgrip strength. The subjects were asked to hold the handle of the dynamometer using the second joints of all fingers except the thumb and pull the handle as hard as they could for 5 sec after adjusting the width of the joints to $90^{\circ}$, while the angle between the body and the stretched arm was maintained at $15^{\circ}$. Two consecutive measurements were recorded, and the maximum value was recorded to the precision of $0.1 \mathrm{~kg}$. The muscular endurance was assessed by performing the sit-up test for $1 \mathrm{~min}$, and the number of sit-ups performed was recorded. In this test, the subjects were asked to lie down with their hands clasped and supporting the head, lift the upper body up till both the elbows touched the knees, and then descend till both the shoulders touched the floor, without using momentum. For flexibility, the sit-and-reach test was used. In this test, the subjects had to sit with stretched knees and upright feet that touched the entire vertical surface of the measuring tool (DW-782, Daewoosports Industry). They then brought their arms together to let the tips of the fingers push the measuring ruler as far as possible and maintain the position for $3 \mathrm{sec}$ while the distance was recorded. Two measurements were recorded, and the maximum value was recorded to the precision of $0.1 \mathrm{~cm}$. The cardiorespiratory fitness was assessed by a 1,200-m running test. The subjects ran on a $1,200-\mathrm{m}$ field track at their maximum speed, and the total elapsed time was recorded to the precision of $0.01 \mathrm{sec}$.

\section{Statistical analysis}

All measured data were represented as means and standard deviations. To analyze the effect of the exercises, a paired sample t-test was performed on the pre- and postexercise values. All statistical processing was performed using IBM SPSS Statistics ver. 25.0 (IBM Co., Armonk, NY, USA), and the significance level ( $\alpha$ ) was set at $P \leq 0.05$. 
Lee JS, et al. • Effect of high-intensity circuit training

Table 3. Changes in the obesity indexes after 4 weeks of high-intensity circuit training

\begin{tabular}{lcccc}
\hline Variable & Before exercise & After exercise & $\Delta$ Score & $P$-value \\
\hline Weight $(\mathrm{kg})$ & $63.48 \pm 9.01$ & $61.50 \pm 9.10$ & $-1.98 \pm 0.54$ & 0.001 \\
BMI $\left(\mathrm{kg} / \mathrm{m}^{2}\right)$ & $25.14 \pm 2.51$ & $24.24 \pm 2.60$ & $-0.90 \pm 0.35$ & 0.001 \\
Body fat $(\%)$ & $30.46 \pm 3.95$ & $29.26 \pm 3.65$ & $-1.20 \pm 0.96$ & 0.003 \\
LBM $(\mathrm{kg})$ & $42.89 \pm 4.80$ & $42.65 \pm 5.08$ & $-1.01 \pm 1.24$ & 0.320 \\
WC $(\mathrm{cm})$ & $76.89 \pm 7.77$ & $75.15 \pm 7.75$ & $-1.74 \pm 1.39$ & 0.003 \\
\hline
\end{tabular}

Values are presented as mean \pm standard deviation.

$\mathrm{BMI}$, body mass index; LBM, lean body mass; WC, waist circumference.

$\Delta$ score represents the difference between the score after and before the exercise program.

Table 4. Changes in irisin and fibroblast growth factor-21 after 4 weeks of high-intensity circuit training

\begin{tabular}{lcccc}
\hline Variable & Before exercise & After exercise & $\Delta$ Score & $P$-value \\
\hline Irisin $(\mu \mathrm{g} / \mathrm{mL})$ & $3.50 \pm 0.77$ & $4.03 \pm 1.18$ & $0.53 \pm 1.15$ & 0.180 \\
FGF-21 $(\mathrm{pg} / \mathrm{mL})$ & $283.06(115.58-415.44)$ & $194.41(76.50-428.07)$ & $-107.84 \pm 237.55$ & 0.182 \\
\hline
\end{tabular}

Values are presented as mean \pm standard deviation or mean (interquartile range).

FGF-21, fibroblast growth factor-21.

$\Delta$ score represents the difference between the score after and before the exercise program.

Table 5. Changes physical fitness after 4 weeks of high-strength circuit training

\begin{tabular}{lcccc}
\hline Variable & Before exercise & After exercise & $\Delta$ Score & $P$-value \\
\hline Handgrip strength $(\mathrm{kg})$ & $26.51 \pm 5.21$ & $27.52 \pm 4.54$ & $1.01 \pm 1.24$ & 0.030 \\
Sit-up (repetition) & $28.4 \pm 7.93$ & $31.10 \pm 6.93$ & $2.70 \pm 3.16$ & 0.024 \\
Sit-and-reach test $(\mathrm{cm})$ & $13.23 \pm 7.38$ & $14.60 \pm 6.46$ & $1.37 \pm 2.46$ & 0.113 \\
1,200 -m running test $(\mathrm{sec})$ & $433.56 \pm 50.64$ & $423.70 \pm 52.10$ & $-9.86 \pm 6.35$ & 0.001
\end{tabular}

Values are presented as mean \pm standard deviation.

$\Delta$ score represents the difference between the score after and before the exercise program.

\section{RESULTS}

\section{Change in physique and body composition}

The changes in the body composition after the 4-week high-intensity CT are shown in Table 3. The postexercise weight showed a significant decrease by approximately $2 \mathrm{~kg}(P<0.001)$, and the obesity indices of WC and BF\% decreased significantly by approximately $1.74 \mathrm{~cm}(P=0.003)$ and $1.2 \%(P=0.003)$, respectively. The muscle mass showed no significant difference between the pre- and postexercise measurements.

\section{Changes in the irisin and FGF-21 levels}

The changes in the irisin and FGF-21 browning factors after the 4-week high-intensity CT are shown in Table 4. After CT, the irisin levels increased by approximately $0.53 \pm 1.15 \mu \mathrm{g} / \mathrm{mL}$ and the FGF-21 levels decreased by approximately $107.84 \pm 237.55$ $\mathrm{pg} / \mathrm{mL}$, but neither showed a significance difference.

\section{Changes in physical fitness}

The changes in the physical fitness after the 4-week high-intensity $\mathrm{CT}$ are shown in Table 5 . There was significant improvement in the handgrip strength test conducted for the muscular strength $(P=0.030), 1$-min sit-up test for the muscular endurance $(P=$ $0.024)$, and $1,200-\mathrm{m}$ running test for the cardiorespiratory fitness $(P<0.001)$. However, no significant difference was found in the sit-and-reach test, the flexibility indicator.

\section{DISCUSSION}

This study aimed to verify the effect of a 4-week high-intensity CT exercise program on the physical fitness, obesity indices, and browning factor levels in inactive female college students. The results showed that the obesity indices significantly decreased after 4-week high-intensity CT, with significant improvements in the physical fitness indicators of muscular strength, muscular endurance, and cardiorespiratory fitness. For the browning factors, the 
irisin levels increased but FGF-21 levels decreased, with no statistical significance.

Previous studies on irisin have reported that the postexercise level of irisin varies depending on the exercise type and intensity and the short-term or long-term duration. In a study conducted on healthy male adults in their 20s, the comparison between high-intensity and low-intensity aerobic exercises inducing the same calorie burn showed that the level of irisin in blood increased by $23 \%$ within $19 \mathrm{hr}$ after performing the high-intensity exercises (Tsuchiya et al., 2014). In a study conducted on obese female adolescents, the comparison between short-term moderate- to high-intensity aerobic exercises and high-intensity exercises performed in intervals showed that the level of irisin in muscular tissues increased significantly after the latter (Archundia-Herrera et al., 2017). Furthermore, a study conducted on healthy male adults reported that after 20 days of high-intensity interval training, the level of irisin mRNA increased (Eaton et al., 2018). Unlike previous studies, our study did not observe a significant change in the irisin levels of inactive female college students performing a 4-week high-intensity CT program, despite showing an increasing trend. Similar results have frequently been reported by studies investigating the effect of training. A review of the relationship between irisin and exercise showed that though the irisin level generally increases after short-term exercises, it does not change after long-term exercises. Similarly, Norheim et al. (2014) reported that the level of irisin in blood after a short-term aerobic exercise significantly increased by approximately 1.2-fold, whereas a 12-week training program in identical subjects did not induce a significant change. In a study by Hecksteden et al. (2013), the group performing aerobic and resistance exercises for 26 weeks showed no significant changes in the irisin level as compared to the control group. In a study conducted on healthy adults of the same age group as in this study, the irisin levels did not show any significant changes after short-term or 3-week long high-intensity resistance training exercises (Fernandez-del-Valle et al., 2018). A review of the irisin level after a short-term exercise reported that the level increased by approximately $15 \%$ on an average (Fox et al., 2018), but a review of the irisin level after a long-term exercise reported no change after the exercise (Qiu et al., 2015). These findings could be due to the rapid half-life of irisin (Hecksteden et al., 2013); the results of our study are also likely to be similarly influenced, as the blood test was performed $72 \mathrm{hr}$ after exercise to avoid the temporary exercise effects and because the blood was refrigerated until the test rather than being tested immediately. Moreover, the irisin level follows a circadian rhythm, such that the lowest level is generally observed at 6 a.m. and the highest level at 9 p.m. (Anastasilakis et al., 2014). The blood collection in this study was performed in the morning for the fasting blood samples. Therefore, future studies investigating the effect of exercise on the irisin level should consider the time of measurement of the irisin level in the study design.

FGF-21, another browning factor like irisin, is known to be produced by various tissues such as the muscular tissue (Fon Tacer et al., 2010). It not only plays a physiological role in the regulation of glucose and lipid metabolism and the browning of the white adipose tissues, but also in the increase in energy consumption (Fisher et al., 2012; Kharitonenkov et al., 2005; Lee et al., 2013). However, compared to irisin, studies on the effect of exercise on FGF-21 are lacking, and the existing studies present inconsistent results. A previous study analyzing the effect of $60 \mathrm{~min}$ of high-intensity cycle ergometer exercise in healthy adults in their 20s reported that the postexercise levels of FGF-21 showed a significant increase (Tanimura et al., 2016). Likewise, in a study by Cuevas-Ramos et al. (2012), a 2-week treadmill exercise reportedly increased the FGF-21 levels significantly in healthy female adults, whereas the overweight individuals showed no change. In this study, the FGF-21 levels after the 4-week high-intensity CT showed an increasing trend, but without statistical significance. These findings coincided with those of the study by Taniguchi et al. (2016), in which a 5-week cycle exercise (3 times a week) was performed. Thus, the relationship between exercise and FGF-21 remains unclear. Nevertheless, a possible cause of the decrease in the FGF-21 levels after exercise could be the influence of insulin and the decrease in the liver lipids. In the study by Taniguchi et al. (2016), the fall in the postexercise level of FGF-21 was reported to have been caused by the fall in the liver lipids, while Cuevas-Ramos et al. (2012) suggested a possible influence of increased insulin after exercise on FGF-21 production. However, the evidence is insufficient, and further studies should be conducted to clearly identify the mechanisms causing the change in the level of FGF-21 after exercise.

This study has several limitations. First, the effect of high-intensity CT was tested after 4 weeks, and further studies should be conducted to evaluate its long-term effect. Second, in the absence of the control group, the dietary intake could not be completely controlled. Nonetheless, it was found that the level of irisin was not influenced by the dietary intake or nutrient composition in a previous study analyzing the determinants of the irisin level (Anastasilakis et al., 2014). Third, objective monitoring of the physical activities performed other than the CT could not be conduct- 
ed. However, the subjects in this study were asked to retain a dietary intake pattern similar to that followed before the study and not perform any other exercises other than the $\mathrm{CT}$ program in this study. The subjects were also interviewed on the current dietary intake and exercise during each session of the CT program.

Despite a relatively short duration of exercise in this study, there were significant improvements in the physical composition and physical fitness, with an increasing trend of the irisin level, which is an adipose tissue browning factor. While the effect of exercise on the browning factors could not be clearly identified against the initial hypothesis, this study may have been significant in providing the evidence for exercise-based health management in a relatively inactive group of female college students.

\section{CONFLICT OF INTEREST}

No potential conflict of interest relevant to this article was reported.

\section{ACKNOWLEDGMENTS}

This work was supported by the 2019 Inje University research grant.

\section{REFERENCES}

Anastasilakis AD, Polyzos SA, Saridakis ZG, Kynigopoulos G, Skouvaklidou EC, Molyvas D, Vasiloglou MF, Apostolou A, KaragiozoglouLampoudi T, Siopi A, Mougios V, Chatzistavridis P, Panagiotou G, Filippaios A, Delaroudis S, Mantzoros CS. Circulating irisin in healthy, young individuals: day-night rhythm, effects of food intake and exercise, and associations with gender, physical activity, diet, and body composition. J Clin Endocrinol Metab 2014;99:3247-3255.

Archundia-Herrera C, Macias-Cervantes M, Ruiz-Muñoz B, Vargas-Ortiz K, Kornhauser C, Perez-Vazquez V. Muscle irisin response to aerobic vs. HIIT in overweight female adolescents. Diabetol Metab Syndr 2017;9:101.

Boström P, Wu J, Jedrychowski MP, Korde A, Ye L, Lo JC, Rasbach KA, Bostrom EA, Choi JH, Long JZ, Kajimura S, Zingaretti MC, Vind BF, Tu H, Cinti S, Højlund K, Gygi SP, Spiegelman BM. A PGC1- $\alpha$-dependent myokine that drives brown-fat-like development of white fat and thermogenesis. Nature 2012;481:463-468.

Coskun T, Bina HA, Schneider MA, Dunbar JD, Hu CC, Chen Y, Moller

$\mathrm{DE}$, Kharitonenkov A. Fibroblast growth factor 21 corrects obesity in mice. Endocrinology 2008;149:6018-6027.
Cuevas-Ramos D, Almeda-Valdés P, Meza-Arana CE, Brito-Córdova G, Gómez-Pérez FJ, Mehta R, Oseguera-Moguel J, Aguilar-Salinas CA. Exercise increases serum fibroblast growth factor 21 (FGF21) levels. PLoS One 2012;7:e38022.

Donaldson AA, Gordon CM. Skeletal complications of eating disorders. Metabolism 2015;64:943-951.

Eaton M, Granata C, Barry J, Safdar A, Bishop D, Little JP. Impact of a single bout of high-intensity interval exercise and short-term interval training on interleukin-6, FNDC5, and METRNL mRNA expression in human skeletal muscle. J Sport Health Sci 2018;7:191-196.

Fernandez-del-Valle M, Short MJ, Chung E, McComb J, Kloiber S, Naclerio F, Larumbe-Zabala E. Effects of high-intensity resistance training on circulating levels of irisin in healthy adults: a randomized controlled trial. Asian J Sports Med 2018;9:e13025.

Fisher FM, Kleiner S, Douris N, Fox EC, Mepani RJ, Verdeguer F, Wu J, Kharitonenkov A, Flier JS, Maratos-Flier E, Spiegelman BM. FGF21 regulates PGC- $1 \alpha$ and browning of white adipose tissues in adaptive thermogenesis. Genes Dev 2012;26:271-281.

Fon Tacer K, Bookout AL, Ding X, Kurosu H, John GB, Wang L, Goetz R, Mohammadi M, Kuro-o M, Mangelsdorf DJ, Kliewer SA. Research resource: comprehensive expression atlas of the fibroblast growth factor system in adult mouse. Mol Endocrinol 2010;24:2050-2064.

Fox J, Rioux BV, Goulet EDB, Johanssen NM, Swift DL, Bouchard DR, Loewen $\mathrm{H}$, Sénéchal M. Effect of an acute exercise bout on immediate post-exercise irisin concentration in adults: a meta-analysis. Scand J Med Sci Sports 2018;28:16-28.

Giralt M, Gavalda-Navarro A. Villarroya F. Fibroblast growth factor-21, energy balance and obesity. Mol Cell Endocrinol 2015;418:66-73.

Hecksteden A, Wegmann M, Steffen A, Kraushaar J, Morsch A, Ruppenthal S, Kaestner L, Meyer T. Irisin and exercise training in humans results from a randomized controlled training trial. BMC Med 2013; 11:235.

Heilbronn LK, Milner KL, Kriketos A, Russell J, Campbell LV. Metabolic dysfunction in anorexia nervosa. Obes Res Clin Pract 2007;1:139-146.

Hojman P, Pedersen M, Nielsen AR, Krogh-Madsen R, Yfanti C, Akerstrom T, Nielsen S, Pedersen BK. Fibroblast growth factor-21 is induced in human skeletal muscles by hyperinsulinemia. Diabetes 2009;58:2797-2801.

Kharitonenkov A, Shiyanova TL, Koester A, Ford AM, Micanovic R, Galbreath EJ, Sandusky GE, Hammond LJ, Moyers JS, Owens RA, Gromada J, Brozinick JT, Hawkins ED, Wroblewski VJ, Li DS, Mehrbod F, Jaskunas SR, Shanafelt AB. FGF-21 as a novel metabolic regulator. J Clin Invest 2005;115:1627-1635.

Kim KH, Kim SH, Min YK, Yang HM, Lee JB, Lee MS. Acute exercise induces FGF21 expression in mice and in healthy humans. PLoS One 


\section{3;8:e63517.}

Klika B, Jordan C. High-intensity circuit training using body weight: maximum results with minimal investment. ACSMs Health Fit J 2013; 17:8-13.

Lackman J, Smith ML, McNeill EB. Freshman college students' reasons for enrolling in and anticipated benefits from a basic college physical education activity course. Front Public Health 2015;24:162.

Lee P, Brychta RJ, Linderman J, Smith S, Chen KY, Celi FS. Mild cold exposure modulates fibroblast growth factor 21 (FGF21) diurnal rhythm in humans: relationship between FGF21 levels, lipolysis, and cold-induced thermogenesis. J Clin Endocrinol Metab 2013;98:E98-E102.

Norheim F, Langleite TM, Hjorth M, Holen T, Kielland A, Stadheim HK, Gulseth HL, Birkeland KI, Jensen J, Drevon CA. The effects of acute and chronic exercise on PGC- $1 \alpha$, irisin and browning of subcutaneous adipose tissue in humans. FEBS J 2014;281:739-749.

Paoli A, Moro T, Marcolin G, Neri M, Bianco A, Palma A, Grimaldi K. High-intensity interval resistance training (HIRT) influences resting energy expenditure and respiratory ratio in non-dieting individuals. J Transl Med 2012;10:237.

Paoli A, Pacelli F, Bargossi AM, Marcolin G, Guzzinati S, Neri M, Bianco A, Palma A. Effects of three distinct protocols of fitness training on body composition, strength and blood lactate. J Sports Med Phys Fitness 2010;50:43-51.

Pedersen BK, Akerstrom TC, Nielsen AR, Fischer CP. Role of myokines in exercise and metabolism. J Appl Physiol 2007;103:1093-1098.

Potthoff MJ, Inagaki T, Satapati S, Ding X, He T, Goetz R, Mohammadi M,
Finck BN, Mangelsdorf DJ, Kliewer SA, Burgess SC. FGF21 induces PGC- $1 \alpha$ and regulates carbohydrate and fatty acid metabolism during the adaptive starvation response. Proc Natl Acad Sci U S A 2009;106: 10853-10858.

Qiu S, Cai X, Sun Z, Schumann U, Zügel M, Steinacker JM. Chronic exercise training and circulating irisin in adults: a meta-analysis. Sports Med 2015;45:1577-1588.

Skidmore BL, Jones MT, Blegen M, Matthews TD. Acute effects of three different circuit weight training protocols on blood lactate, heart rate, and rating of perceived exertion in recreationally active women. J Sports Sci Med 2012;11:660-668.

Taniguchi H, Tanisawa K, Sun X, Kubo T, Higuchi M. Endurance exercise reduces hepatic fat content and serum fibroblast growth factor $21 \mathrm{lev}$ els in elderly men. J Clin Endocrinol Metab 2016;101:191-198.

Tanimura Y, Aoi W, Takanami Y, Kawai Y, Mizushima K, Naito Y, Yoshikawa T. Acute exercise increases fibroblast growth factor 21 in metabolic organs and circulation. Physiol Rep 2016;4:e12828.

Tsuchiya Y, Ando D, Goto K, Kiuchi M, Yamakita M. High-intensity exercise causes greater irisin response compared with low-intensity exercise under similar energy consumption. Tohoku J Exp Med 2014;233: 135-140.

Xiong XQ, Chen D, Sun HJ, Ding L, Wang JJ, Chen Q, Li YH, Zhou YB, Han Y, Zhang F, Gao XY, Kang YM, Zhu GQ. FNDC5 overexpression and irisin ameliorate glucose/lipid metabolic derangements and enhance lipolysis in obesity. Biochim Biophys Acta 2015;1852:1867-1875. 